\title{
Erratum to: Fe Oxides on Ag Surfaces: Structure and Reactivity
}

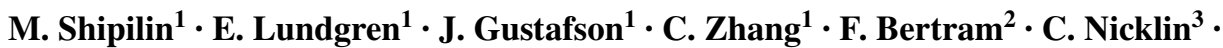

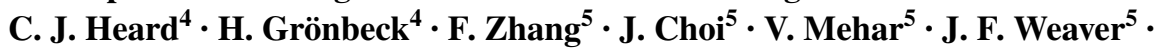 \\ L. R. Merte $^{1}$
}

Published online: 30 May 2017

(C) Springer Science+Business Media New York 2017

\section{Erratum to: Top Catal \\ DOI 10.1007/s11244-016-0714-8}

An author on this paper was misidentified as "V.J.F. Weaver." The correction was made to the paper correctly listing "J.F. Weaver" in the author group.

The online version of the original article can be found under doi:10.1007/s11244-016-0714-8.

M. Shipilin

mikhail.shipilin@sljus.lu.se

1 Division of Synchrotron Radiation Research, Lund

University, Box 118, 22100 Lund, Sweden

2 DESY Photon Science, Notkestr. 85, 22607 Hamburg, Germany

3 Diamond Light Source, OX11 0DE Didcot, Oxfordshire, UK

4 Chalmers University of Technology, 41296 Göteborg, Sweden

5 Department of Chemical Engineering, University of Florida, Gainesville, FL 32611, USA 